\title{
Practical Robust Positive Invariance for Large-Scale Discrete Time Systems
}

\author{
by \\ Saša V. Raković, Benjamin Kern and Rolf Findeisen
}

August 2, 2011

Technical Report IFAT-SYS 1/2011

Otto-von-Guericke University Magdeburg

Institute for Automation Engineering

Universitätsplatz 2

39106 - Magdeburg

Germany 


\title{
Practical Robust Positive Invariance for Large-Scale Discrete Time Systems
}

\author{
August 2, 2011
}

\begin{abstract}
This note introduces practical set invariance notions for physically interconnected, discrete-time systems, subject to additive but bounded disturbances. We offer a concept which allows us to investigate invariance properties for each of the subsystems separately and, hence, to study invariance properties of the overall system in a sensible and decentralized way. The analysis part makes use of stability and invariance notions of properly specified set-dynamics. The developed approach provides a decentralized, non-conservative and computationally tractable way to study desirable robust positive invariance and stability notions for the corresponding set-dynamics and, in turn, for the overall system as well as to guarantee safe and independent operation of the constituting subsystems. These desirable and strong properties are inherited, under mild assumptions, from the classical stability and invariance properties of the associated vector-valued dynamics which capture in a simple but appropriate and non-conservative way the dynamical behavior induced by the underlying set-dynamics of interest.
\end{abstract}

Large-scale Systems, Interconnected Systems, Set Invariance, Stability, Constraints, Uncertainty, Set-Dynamics.

\section{Introduction}

The analysis of large-scale systems offers many challenging system-theoretic problems. A centralized solution methodology is, from the analytical point of view, the preferred option for addressing the corresponding issues; however, it is often not practicable due to, for instance, computational complexity, bandwidth limitations or economical considerations. A modular approach is usually employed to circumvent these problems; the core idea of this approach is to decompose the large-scale system into smaller but interconnected subsystems. The main advantage is obvious, since within this setting, a number of smaller-dimensional subsystems needs to be analyzed and their system-theoretic properties, under suitable conditions on the interconnection terms, allow for the corresponding system-theoretic properties of the overall system to be guaranteed. For an in depth overview of control synthesisand analysis-methods for continuous-time decentralized systems, see the important works $[18,19,4]$ and references therein.

In general, the modular analysis and controller synthesis of interconnected systems benefits greatly from the use of vector Lyapunov-functions [5, 9]. Essentially, instead of a single Lyapunov-function, several Lyapunov-functions and their dynamics are used to analyze stability properties of the overall system dynamics. Motivated by this notion and the recent results on set invariance under state and output feedback utilizing set-dynamics [1, 2], in [12] a different set invariance notion for interconnected systems was introduced and investigated. In particular, it was shown in [12], that several "dynamically interconnected positively invariant sets" for the constituting subsystems lead to similar invariance properties for the whole system, providing that an appropriately designed dynamics of suitably parametrized sets is stable. The approach of [12] allowed for the non-conservative use of the well-established set invariance concepts applied to the subsystems which are lower-dimensional, and, hence, it avoided the extremely demanding and possibly intractable computation of a single positively invariant set for the higher-dimensional overall system. The practical positive invariance notion introduced in [12] allowed for the independent initialization and safe operation of the constituting subsystems as well as non-conservative handling of the underlying interconnection effects via suitably designed dynamical behavior of the employed collection of positively invariant sets.

The prime objective of this note is to generalize the set invariance and stability notions of [12] in order to provide flexible and practicable notions allowing for safe, stable and independent operation of all subsystems (and, in turn, the overall system) despite the presence of constraints, restrictions on the amount of information available locally (this "informational restriction" is inevitably induced by the decentralization of the original system) as well as the presence of additive but bounded disturbances.

\section{Paper Structure:}

Section 2 provides preliminaries and outlines the general problem setting. Section 3 reviews briefly practical set invariance for a family of sets in the nominal case. Section 4 discusses the extension of practical set invariance to the 
robust case and delivers the main contribution of this work. Section 5 addresses the corresponding convergence and stability issues. Section 6 comments on computational aspects and delivers an illustrative example. Section 7 closes this note with a few concluding remarks.

\section{Basic Nomenclature and Definitions:}

The sets of positive, non-negative integers and non-negative reals are denoted by $\mathbb{N}_{+}, \mathbb{N}$ and $\mathbb{R}_{+}$. For each positive integer $q \in \mathbb{N}_{+}, \mathbb{N}_{[1: q]}:=\{1,2, \ldots, q\}$. For $r \in \mathbb{N}_{[1: q]}$, we denote $\mathbb{N}_{(q, r)}:=\{1, \ldots, r-1, r+1, \ldots, q\}=\mathbb{N}_{[1: q]} \backslash\{r\}$. A set $X$ is non-trivial, if it is a proper, non-empty subset of $\mathbb{R}^{n}$ and not a singleton. Given two sets $X, Y$ in $\mathbb{R}^{n}$, the Minkowski set addition is defined by $X \oplus Y:=\{x+y: x \in X, y \in Y\}$. For a set sequence $\left\{X_{i} \subset \mathbb{R}^{n}\right\}_{i=a}^{b}$, where $a, b \in \mathbb{N}, b>a$, we define $\bigoplus_{i=a}^{b} X_{i}:=X_{a} \oplus \cdots \oplus X_{b}$. Given a set $X$ in $\mathbb{R}^{n}$ and a real matrix $M$ of compatible dimension (possibly a scalar), we define the image of $X$ under $M$ by $M X:=\{M x: x \in X\}$ and the preimage of $X$ under $M$ by $M^{-1} X:=\{x: M x \in X\}$. For any matrix $M \in \mathbb{R}^{n \times n}, \rho(M)$ denotes the largest absolute value of its eigenvalues. A set $X \subset \mathbb{R}^{n}$ is a $C$-set, if it is compact, convex and contains the origin. A set $X \subset \mathbb{R}^{n}$ is a proper $C$-set $(P C$-set) if it is a $C$-set, which contains the origin in its (non-empty) interior. A collection of sets $\left\{X_{i} \subset \mathbb{R}^{n}: i \in \mathbb{N}_{[1: q]}\right\}$ is a $P C$-collection if each $X_{i}$ is a $P C$-set. A set $X \subseteq \mathbb{R}^{n}$ is symmetric, with respect to the origin in $\mathbb{R}^{n}$, if $X=-X$. A polyhedron is the (convex) intersection of a finite number of open and/or closed half-spaces and a polytope is the closed and bounded polyhedron. The family of all subsets of $\mathbb{R}^{n}$ is denoted by $2^{n}$. The family of non-empty compact subsets in $\mathbb{R}^{n}$ is denoted by $\operatorname{Com}\left(\mathbb{R}^{n}\right)$.

For any closed convex set $Y$ in $\mathbb{R}^{n}$, the function specified by:

$$
s(Y, x):=\sup _{y}\left\{x^{T} y: y \in Y\right\} \text { for } x \in \mathbb{R}^{n},
$$

is called the support function. Given a proper $C$-set $L$ in $\mathbb{R}^{n}$, the function given by:

$$
g(L, x):=\min _{\mu \geq 0}\{\mu: x \in \mu L\} \text { for } x \in \mathbb{R}^{n},
$$

is called the gauge (Minkowski) function. If $L \subset \mathbb{R}^{n}$ is a symmetric, proper $C$-set, then the gauge-function $g(L, \cdot)$ also induces the vector $L$-norm, given by

$$
|x|_{L}:=g(L, x) \text { for } x \in \mathbb{R}^{n} .
$$

For $X \in \operatorname{Com}\left(\mathbb{R}^{n}\right)$ and $Y \in \operatorname{Com}\left(\mathbb{R}^{n}\right)$, the Hausdorff semi-distance and the Hausdorff distance of $X$ and $Y$ are given by:

$$
\begin{aligned}
h(L, X, Y) & :=\min _{\alpha}\{\alpha: X \subseteq Y \oplus \alpha L, \alpha \geq 0\} \text { and } \\
H(L, X, Y) & :=\max \{h(L, X, Y), h(L, Y, X)\},
\end{aligned}
$$

where $L$ is a given, symmetric, proper $C$-set in $\mathbb{R}^{n}$.

\section{Preliminaries}

Throughout this note we consider a set of $N$ discrete-time, time-invariant, linear interconnected systems given by:

$$
\forall i \in \mathbb{N}_{[1: N]}, x_{i}^{+}=A_{(i, i)} x_{i}+\sum_{j \in \mathbb{N}_{(N, i)}} A_{(i, j)} x_{j}+w_{i},
$$

where $\forall i \in \mathbb{N}_{[1: N]}, x_{i} \in \mathbb{R}^{n_{i}}$ is the current state of the $i^{t h}$ subsystem, $A_{(i, i)} \in \mathbb{R}^{n_{i} \times n_{i}}$ is the state transition matrix of the $i^{\text {th }}$ subsystem and $w_{i} \in \mathbb{W}_{i}$ denotes a disturbance acting on the $i^{\text {th }}$ subsystem. The current overall state is given by $x=\left(x_{1}, x_{2}, \ldots, x_{N}\right) \in \mathbb{R}^{n}$, with $n=\sum_{i \in \mathbb{N}_{[1: N]}} n_{i}$. For each $i \in \mathbb{N}_{[1: N]}$ and $j \in \mathbb{N}_{(N, i)}$, the $i^{t h}$ subsystem is affected by the $j^{\text {th }}$ subsystem via the linear, physical interconnections specified by matrices $A_{(i, j)} \in \mathbb{R}^{n_{i} \times n_{j}}$. The states $x_{i}$ and disturbances $w_{i}$ are subject to hard constraints:

$$
\forall i \in \mathbb{N}_{[1: N]}, x_{i} \in \mathbb{X}_{i} \text { and } w_{i} \in \mathbb{W}_{i}
$$

In this note we work under the standard assumption:

Assumption 2.1 For all $i \in \mathbb{N}_{[1: N]}$,:

(i) the sets $\mathbb{X}_{i}$ are proper $C$-sets in $\mathbb{R}^{n_{i}}$, and,

(ii) the sets $\mathbb{W}_{i}$ are $C$-sets in $\mathbb{R}^{n_{i}}$. 
Remark 2.1 Throughout this note we work in the autonomous setting due to space limitations. However, the methods presented here can be easily extended to a much broader setup involving decision making processes/control syntheses for each of the subsystems (where each local decision maker utilizes both the local as well as global information patterns from the other subsystems). Nevertheless, the invoked setting is sufficiently broad to allow the investigation of practical robust positive invariance issues relevant for decentralized control problems of large-scale systems. Furthermore, this setting captures the commonly studied case [18, 19, 4] in which each decision maker $i$ uses local state information of the $i^{\text {th }}$ subsystem (i.e. the local state $x_{i}$ ) and is allowed to employ the linear, static feedback $K_{i} x_{i}$, where $K_{i}$ is a matrix of compatible dimension. Clearly, if the open-loop structure of the constitution subsystems is given by

$$
\forall i \in \mathbb{N}_{[1: N]}, x_{i}^{+}=\bar{A}_{(i, i)} x_{i}+B_{i} u_{i}+\sum_{j \in \mathbb{N}_{(N, i)}} A_{(i, j)} x_{j}+w_{i},
$$

where $u_{i} \in \mathbb{R}^{m_{i}}$ and $B_{i} \in \mathbb{R}^{n_{i} \times m_{i}}$, then setting $A_{(i, i)}=\left(\bar{A}_{(i, i)}+B_{i} K_{i}\right)$ we are within the considered setting. The interested reader is also referred to [12] for a more detailed discussion.

The prime objective of this note is to introduce a non-conservative and practical robust positive invariance notion for the set of $N$ discrete-time, time-invariant, linear interconnected systems given in (1). This naturally leads to the standard concept of robust positive invariance which is now recalled briefly, cf. $[3,6,7]$ :

Definition 2.1 $A$ set $\Omega$ is said to be robust positively invariant for the system $x^{+}=A x+w$ with the constraint set $\mathbb{X}$ and disturbance set $\mathbb{W}$, if and only if $\Omega \subseteq \mathbb{X}$ and for all $x \in \Omega$ and $w \in \mathbb{W}$ it holds that $A x+w \in \Omega$ (i.e. $A \Omega \oplus \mathbb{W} \subseteq \Omega$ ).

Evidently, the most obvious way to obtain robust positive invariant sets for the set of subsystems defined in (1) is to consider the augmented form describing the overall system and given by:

$$
x^{+}=A x+w,
$$

where $x=\left(x_{1}, x_{2}, \ldots, x_{N}\right) \in \mathbb{R}^{n}$ is the overall current state, $w=\left(w_{1}, w_{2}, \ldots, w_{N}\right) \in \mathbb{R}^{n}$ is the overall current disturbance and $A \in \mathbb{R}^{n \times n}$ is the state transition matrix of the overall system which is composed from the matrices in $A_{(i, j)}$ appearing in (1). The overall state $x=\left(x_{1}, x_{2}, \ldots, x_{N}\right) \in \mathbb{R}^{n}$ and disturbance $w=\left(w_{1}, w_{2}, \ldots, w_{N}\right) \in \mathbb{R}^{n}$ are, in view of $(2)$ subject to hard constraints:

$$
\begin{aligned}
& x \in \mathbb{X} \text { and } w \in \mathbb{W}, \text { where, } \mathbb{X}:=\mathbb{X}_{1} \times \mathbb{X}_{2} \cdots \times \mathbb{X}_{N}, \text { and }, \\
& \mathbb{W}:=\mathbb{W}_{1} \times \mathbb{W}_{2} \cdots \times \mathbb{W}_{N} .
\end{aligned}
$$

Indeed, the theory of the maximal and minimal robust positively invariant sets for the system (3) and constraint sets (4) is well studied and understood $[3,8,6,1,7]$. Furthermore, the characterization and computation of the inner and outer robust positively invariant approximations of the maximal and minimal robust positively invariant sets, respectively, for the system (3) and constraint sets (4) is also well understood [13, 10, 1, 11]. Although the algorithmic methods for the computation of the maximal and minimal robust positively invariant sets (as well as their robust positively invariant approximations) for the system (3) and constraint sets (4) exist, the corresponding computational procedures are severely limited by the dimension of the considered problem (rendering, in turn, the standard approaches inapplicable to the case of large-scale systems). Furthermore, the application of the classical robust positive invariance notions yields a robust positively invariant set for the augmented system (3) and constraint sets (4) and, hence, does not permit, in general, for the independent initialization and operation of the constituting subsystems specified in (1) within the corresponding robust positively invariant set. This is due to the fact that once a robust positively invariant set for the overall system (3) and constraint sets (4) has been detected and computed, there will always be, in general, a strict dependence between the local states $x_{i}$ of the different subsystems which in turn does not allow for the independent initialization of each of the processes. This conflicts with our main objective, namely to obtain a robust positive invariance notion which permits the treatment of the large-scale problems in a structured way and, in addition, provides guidelines on how the independent initialization and safe operation, under physical interconnections and disturbances, can be achieved.

Since the approach proposed in this note follows closely the approach of [12] relevant for the nominal case (i.e. in the absence of the additive disturbances), we provide in the next section an overview of the basic terminology in a simplified form.

\section{Practical Positive Invariance}

In [12], we considered the nominal case, namely systems of the form

$$
\forall i \in \mathbb{N}_{[1: N]}, \bar{x}_{i}^{+}=A_{(i, i)} \bar{x}_{i}+\sum_{j \in \mathbb{N}_{(N, i)}} A_{(i, j)} \bar{x}_{j}
$$


where $\forall i \in \mathbb{N}_{[1: N]}, j \in \mathbb{N}_{(N, i)}, \bar{x}_{i} \in \mathbb{R}^{n_{i}}, A_{(i, i)} \in \mathbb{R}^{n_{i} \times n_{i}}, A_{(i, j)} \in \mathbb{R}^{n_{i} \times n_{j}}$. The local states $\bar{x}_{i}, i \in \mathbb{N}_{[1: N]}$ are subject to hard constraints:

$$
\forall i \in \mathbb{N}_{[1: N]}, \bar{x}_{i} \in \mathbb{X}_{i}
$$

where the constraint sets $\mathbb{X}_{i}, i \in \mathbb{N}_{[1: N]}$ satisfy Assumption $2.1(i)$. The main role in the examination of practical set invariance is played by the induced, independent, set-dynamics specified, for all $i \in \mathbb{N}_{[1: N]}$, by:

$$
\begin{aligned}
& \bar{X}_{i}^{+}=F_{i}(\bar{X}), \text { with } \\
& F_{i}(\bar{X}):=A_{(i, i)} \bar{X}_{i} \oplus \bigoplus_{j \in \mathbb{N}_{[1: N]}} A_{(i, j)} \bar{X}_{j},
\end{aligned}
$$

where $\bar{X}=\left(\bar{X}_{1}, \bar{X}_{2}, \ldots, \bar{X}_{N}\right) \in 2^{\mathbb{R}^{n_{1}}} \times 2^{\mathbb{R}^{n_{2}}} \times \cdots \times 2^{\mathbb{R}^{n_{N}}}$. Within this setting, the exact set invariance problem requires the characterization and determination of an invariant family of sets. The complexity of the exact set invariance problem is in [12] alleviated by the introduction of practical positive invariance notion. Namely, the notion that utilizes a parameterized family of $\operatorname{sets} \mathcal{S}(\mathbb{S}, \Theta)$ specified by:

$$
\mathcal{S}(\mathbb{S}, \Theta):=\left\{\left(\theta_{1} S_{1}, \theta_{2} S_{2}, \ldots, \theta_{N} S_{N}\right): \theta \in \Theta\right\}
$$

where $\mathbb{S}$ is a prescribed collection of sets given by:

$$
\mathbb{S}:=\left\{S_{i}: i \in \mathbb{N}_{[1: N]}\right\},\left(S_{1}, \ldots, S_{N}\right) \in 2^{\mathbb{R}^{n_{1}}} \times \ldots \times 2^{\mathbb{R}^{n_{N}}},
$$

and $\Theta \subseteq \mathbb{R}_{+}^{N}$ is a suitably defined set. More specifically, it was shown in [12] that, under Assumption 2.1 ( $i$ ), the collection of sets $\mathbb{S}$ can be taken to be a $P C$-collection of sets (without loss of generality in an adequate sense). Simple linear functions specified, for all $i \in \mathbb{N}_{[1: N]}$, by:

$$
\begin{aligned}
& \theta_{i}^{+}=\sum_{j \in \mathbb{N}_{[1: N]}} \mu_{(i, j)} \theta_{j}, \text { with } \forall(i, j) \in \mathbb{N}_{[1: N]} \times \mathbb{N}_{[1: N]}, \\
& \mu_{(i, j)}:=\min _{\mu \geq 0}\left\{\mu: A_{(i, j)} S_{j} \subseteq \mu S_{i}\right\}
\end{aligned}
$$

are associated with the parameterized family of sets $\mathcal{S}(\mathbb{S}, \Theta$ ) (which is itself induced from a prescribed $P C$-collection of sets $\mathbb{S}$ and the suitable set $\Theta$ ). These linear functions capture the dynamics of the scaling factors $\theta=\left(\theta_{1}, \theta_{2}, \ldots, \theta_{N}\right)$ which takes the form of a linear autonomous discrete-time system:

$$
\theta^{+}=M \theta
$$

where $\theta \in \mathbb{R}_{+}^{N}$ is the current value of the scaling factors, $\theta^{+} \in \mathbb{R}_{+}^{N}$ is the successor value of the scaling factors and $M \in \mathbb{R}_{+}^{N \times N}$ is the matrix composed from the scalars $\mu_{(i, j)} \in \mathbb{R}_{+},(i, j) \in \mathbb{N}_{[1: N]} \times \mathbb{N}_{[1: N]}$ defined in (10). We note that, by construction, given a current value of the scaling factors $\theta \in \mathbb{R}_{+}^{N}$ it is guaranteed that, for all $i \in \mathbb{N}_{[1: N]}$, it holds that $F_{i}\left(\left(\theta_{1} S_{1}, \theta_{2} S_{2}, \ldots, \theta_{N} S_{N}\right)\right) \subseteq \theta_{i}^{+} S_{i}$. The main idea in [12] was to avoid the use of the exact induced, independent, set-dynamics (7) and instead utilize the outer-bounding approximate set-dynamics restricted to the parameterized family of sets $\mathcal{S}(\mathbb{S}, \Theta)$; this, in turn, leads to computationally practicable set invariance notions. The dynamical behavior of the outer-bounding approximate set-dynamics is fully characterized by the simple $\theta$-dynamics given by (11) which are standard vector-valued dynamics. The satisfaction of state constrains (6) is enforced by imposing the constraints on the scalings $\theta$ as specified by:

$$
\Theta_{0}:=\left\{\theta \in \mathbb{R}_{+}^{N}: \forall i \in \mathbb{N}_{[1: N]}, \theta_{i} S_{i} \subseteq \mathbb{X}_{i}\right\}
$$

The set $\Theta$ appearing in the parameterization of the family of sets $\mathcal{S}(\mathbb{S}, \Theta)$ is taken to be a positively invariant set (potentially the maximal positively invariant set) for the $\theta$-dynamics and constraint set $\Theta_{0}$, i.e. the set $\Theta$ satisfies:

$$
\Theta \subseteq \Theta_{0} \text { and } M \Theta \subseteq \Theta
$$

The notion of practical positive invariance as introduced and analyzed in [12] is specified by the following definition:

Definition 3.1 A family of sets $\mathcal{S}(\mathbb{S}, \Theta)$ specified in (8) with $\mathbb{S}$ given by (9) and $\Theta \subseteq \mathbb{R}_{+}^{N}$ is said to be a positively invariant family of sets for the system (5) and constraint set $(6)$, if for all $i \in \mathbb{N}_{[1: N]}$ and all $\left(\theta_{1} S_{1}, \theta_{2} S_{2}, \ldots, \theta_{N} S_{N}\right) \in$ $\mathcal{S}(\mathbb{S}, \Theta)$ it holds that:

$$
\begin{aligned}
& \theta_{i} S_{i} \subseteq \mathbb{X}_{i}, F_{i}\left(\left(\theta_{1} S_{1}, \theta_{2} S_{2}, \ldots, \theta_{N} S_{N}\right)\right) \subseteq \theta_{i}^{+} S_{i}, \text { and } \\
& \left(\theta_{1}^{+} S_{1}, \theta_{2}^{+} S_{2}, \ldots, \theta_{N}^{+} S_{N}\right) \in \mathcal{S}(\mathbb{S}, \Theta)
\end{aligned}
$$


It was demonstrated in [12] that, under Assumption $2.1(i)$ and when $\mathbb{S}$ is a $P C$-collection of sets, the sufficient condition for the existence of a non-trivial positively invariant family of sets for the interconnected system (5) and constraint set (6) is the strict stability of the matrix $M \in \mathbb{R}_{+}^{N \times N}$ inducing the scaling dynamics in (11). In fact, it was also shown in [12] that, under these assumptions, the system (5) can be initiated and operated in an independent fashion while guaranteeing the constraint satisfaction of the overall system as well as stability of the origin with the well-defined domain of attraction (which is induced from the collection of sets $\mathbb{S}$ and the corresponding positively invariant set $\Theta$ ). For a more comprehensive overview of the corresponding notion, its system-theoretic implications and the underlying computational issues we refer the interested reader to [12].

The framework of [12] offered a theoretically sensible, non-conservative and computationally practicable set invariance notion for decentralized systems. It is therefore of great theoretical interest as well as of practical relevance to generalize the corresponding framework in order to address the robust case. This note, therefore, aims to utilize ideas of [12] and provide the practical robust positive invariance notions which lead to non-conservative and computationally practicable set invariance notions for decentralized systems subject to additive but bounded disturbances.

\section{Practical Robust Positive Invariance}

As in the disturbance free case, the requirement for the independent operation of the set of $N$ subsystems affected by the additive uncertainty as specified in (1) leads naturally to induced, independent, set-dynamics given for all $i \in \mathbb{N}_{[1: N]}$ and all $X=\left(X_{1}, X_{2}, \ldots, X_{N}\right) \in 2^{\mathbb{R}^{n_{1}}} \times 2^{\mathbb{R}^{n_{2}}} \times \ldots \times 2^{\mathbb{R}^{n_{N}}}$, by

$$
X_{i}^{+}=F_{i}(X, \mathbb{W})
$$

where $\mathbb{W}=\mathbb{W}_{1} \times \mathbb{W}_{2} \times \ldots \mathbb{W}_{N}$ and

$$
F_{i}(X, \mathbb{W})=A_{(i, i)} X_{i} \oplus \bigoplus_{j \in \mathbb{N}_{(N, i)}} A_{(i, j)} X_{j} \oplus \mathbb{W}_{i}
$$

Within the framework of set-dynamics (15)-(16) several robust positive invariance notions can be considered. In this sense, a potentially interesting alternative is to look for a robust positively invariant set of a particular form consistent with the decentralized form of the overall system. Namely, to aim for the characterization and computation of a robust positively invariant set $\Omega$ taking the form $\Omega=\Omega_{1} \times \Omega_{2} \times \ldots \times \Omega_{N}$ where, for all $i \in \mathbb{N}_{[1: N]}, \Omega_{i} \subseteq \mathbb{R}^{n_{i}}$ and the sets $\Omega_{i}$ are detected separately and computed locally. Within this setting, a possible notion of robust positive invariance is reflected via the following definition:

Definition $4.1 A$ collection of sets $\Omega:=\left\{\Omega_{i}: i \in \mathbb{N}\right\}$, is a robust positively invariant collection of sets for the system (1) and the constraint sets (2), if and only if, for all $i \in \mathbb{N}_{[1: N]}$ it holds that

$$
\begin{aligned}
& \Omega_{i} \subseteq \mathbb{X}_{i} \text { and } \\
& F_{i}\left(\left(\Omega_{1}, \Omega_{2}, \ldots, \Omega_{N}\right), \mathbb{W}\right) \subseteq \Omega_{i} .
\end{aligned}
$$

However, the conditions (17) in Definition 4.1 are, in general case, rather strong and lead to restrictive and unrealistic requirements for the existence of a collection of robust positively invariant sets. More specifically, the condition (17b) is not fully compatible with the induced, independent, set-dynamics specified in (15)-(16) as does not take appropriately into account the underlying set-dynamics. A non-naive and exact robust positive notion is possible but it requires the utilization of a suitable family of sets as specified by:

Definition 4.2 A family of sets $\mathcal{X} \subseteq 2^{\mathbb{R}^{n_{1}}} \times 2^{\mathbb{R}^{n_{2}}} \times \ldots 2^{\mathbb{R}^{n_{N}}}$ is said to be a robust positively invariant family of sets for the system (1) and constraint sets (2) if and only if, for all $X=\left(X_{1}, X_{2}, \ldots, X_{N}\right) \in \mathcal{X}$ and all $i \in \mathbb{N}_{[1: N]}$ it holds that:

$$
\begin{aligned}
& X_{i} \subseteq \mathbb{X}_{i}, \\
& F_{i}\left(\left(X_{1}, X_{2}, \ldots, X_{N}\right), \mathbb{W}\right) \subseteq X_{i}^{+}, \text {and }, \\
& X^{+}=\left(X_{1}^{+}, X_{2}^{+}, \ldots, X_{N}^{+}\right) \in \mathcal{X}
\end{aligned}
$$

The concept of robust positively invariant family of sets in Definition 4.2 is fully compatible with the induced, independent, set-dynamics specified in (15)-(16) and allows us to capture appropriately the physical interconnection effects by directly relating them to the dynamical change of the sets $X \in \mathcal{X}$. From a conceptual point of view, Definition 4.2 provides a framework, in which the theoretical analysis can be carried out quite conveniently. However, our equally important aim is to provide computationally tractable robust positive invariance notions and to this end we proceed to analyze a sensible trade-off between naive and conservative notions of Definition 4.1 and general 
and flexible notions of Definition 4.2. The main idea is to reach a reasonable trade-off by considering, as in the disturbance free case, a parameterized family of sets $\mathcal{S}(\mathbb{S}, \Theta)$ specified by:

$$
\mathcal{S}(\mathbb{S}, \Theta):=\left\{\left(\theta_{1} S_{1}, \theta_{2} S_{2}, \ldots, \theta_{N} S_{N}\right): \theta \in \Theta\right\}
$$

where $\theta=\left(\theta_{1}, \theta_{2}, \ldots, \theta_{N}\right) \in \mathbb{R}_{+}^{N}, \Theta \subseteq \mathbb{R}_{+}^{N}$ and, for all $i \in \mathbb{N}_{[1: N]}, S_{i} \in 2^{\mathbb{R}^{n_{i}}}$, by invoking the following concept of practical robust positive invariance:

Definition 4.3 Given a collection of sets $\mathbb{S}=\left\{S_{i}: i \in \mathbb{N}_{[1: N]}\right\}$ with $\left(S_{1}, S_{2}, \ldots, S_{N}\right) \in 2^{\mathbb{R}^{n_{1}}} \times 2^{\mathbb{R}^{n_{2}}} \times \ldots 2^{\mathbb{R}_{N}}$ and a set $\Theta \subseteq \mathbb{R}_{+}^{N}$, the family of sets $\mathcal{S}(\mathbb{S}, \Theta)$ specified by (19) is said to be a practical robust positively invariant family of sets for the system (1) and the constraint sets (2) if and only if, for all $i \in \mathbb{N}_{[1: N]}$ and all $\left(\theta_{1} S_{1}, \theta_{2} S_{2}, \ldots, \theta_{N} S_{N}\right) \in$ $\mathcal{S}(\mathbb{S}, \Theta)$, it holds that:

$$
\begin{aligned}
& \theta_{i} S_{i} \subseteq \mathbb{X}_{i}, \\
& F_{i}\left(\left(\theta_{1} S_{1}, \theta_{2} S_{2}, \ldots, \theta_{N} S_{N}\right), \mathbb{W}\right) \subseteq \theta_{i}^{+} S_{i}, \text { and }, \\
& \left(\theta_{1}^{+} S_{1}, \theta_{2}^{+} S_{2}, \ldots, \theta_{N}^{+} S_{N}\right) \in \mathcal{S}(\mathbb{S}, \Theta) .
\end{aligned}
$$

The main rationale behind the practical robust positive invariance notions of Definition 4.3 is to restrict the exact set-dynamics (15)-(16) to a suitable parameterized family of sets $\mathcal{S}(\mathbb{S}, \Theta)$ as specifed in (19) and employ, for computational simplicity, outer-bounding set-dynamics induced from the dynamics of the scaling factors $\theta$. The basic assumption on the collection of sets $\mathbb{S}=\left\{S_{i}: i \in \mathbb{N}_{[1: N]}\right\}$ is, as in the nominal case [12], invoked without loss of generality due to Assumption 2.1:

Assumption 4.1 The collection of sets $\mathbb{S}=\left\{S_{i}: i \in \mathbb{N}_{[1: N]}\right\}$ is a PC-collection of sets.

The one-step set-dynamics condition (18b) in the exact robust positive invariance notions of Definition 4.2 is replaced by a computationally simpler condition (20b) in the practical robust positive invariance notions of Definition 4.3. The requirement (20b) motivates the introduction of a suitable collection of functions $\left\{\mu_{i}(\cdot): i \in \mathbb{N}_{[1: N]}\right\}$ with $\mu_{i}(\cdot) \mathbb{R}_{+}^{N} \rightarrow \mathbb{R}_{+}$capturing the dynamics of the scaling factors $\theta$. The optimal selection of the collection of functions $\left\{\mu_{i}(\cdot): i \in \mathbb{N}_{[1: N]}\right\}$ with $\mu_{i}(\cdot) \mathbb{R}_{+}^{N} \rightarrow \mathbb{R}_{+}$, in the sense of the tightness of the involved set inclusions, is to consider the exact functions $\mu_{i}^{e}(\cdot)$ given, for all $i \in \mathbb{N}_{[1: N]}$ and all $\theta \in \mathbb{R}_{+}^{N}$, by:

$$
\mu_{i}^{e}(\theta):=\min _{\mu \geq 0}\left\{\mu: \bigoplus_{j \in \mathbb{N}_{[1: N]}} \theta_{j} A_{(i, j)} S_{j} \oplus \mathbb{W}_{i} \subseteq \mu S_{i}\right\}
$$

The relevant topological properties of the collection of exact functions $\left\{\mu_{i}^{e}(\cdot): i \in \mathbb{N}_{[1: N]}\right\}$ are summarized by:

Proposition 4.1 Suppose Assumptions 2.1 and 4.1 hold. Then, for all $i \in \mathbb{N}_{[1: N]}$, the functions $\mu_{i}^{e}(\cdot): \mathbb{R}_{+}^{N} \rightarrow \mathbb{R}_{+}$ specified in (21) are convex and continuous functions.

Albeit the collection of exact functions $\left\{\mu_{i}^{e}(\cdot): i \in \mathbb{N}_{[1: N]}\right\}$ enjoys interesting properties established in Proposition 4.1 , the practicability motivates the utilization of the collection of simpler, affine functions $\left\{\mu_{i}(\cdot): i \in \mathbb{N}_{[1: N]}\right\}$ specified, for all $i \in \mathbb{N}_{[1: N]}$ and all $\theta \in \mathbb{R}_{+}^{N}$, by:

$$
\mu_{i}(\theta):=\sum_{j \in \mathbb{N}_{[1: N]}} \mu_{(i, j)} \theta_{j}+\alpha_{i}
$$

where, for each $i$ and $j$,

$$
\begin{aligned}
& \mu_{(i, j)}:=\min _{\mu \geq 0}\left\{\mu: A_{(i, j)} S_{j} \subseteq \mu S_{i}\right\}, \text { and }, \\
& \alpha_{i}:=\min _{\alpha \geq 0}\left\{\alpha: \mathbb{W}_{i} \subseteq \alpha S_{i}\right\} .
\end{aligned}
$$

Clearly, by construction, it follows that, for all $i \in \mathbb{N}_{[1: N]}$ and all $\theta \in \mathbb{R}_{+}^{N}$, we have $0 \leq \mu_{i}^{e}(\theta) \leq \mu_{i}(\theta)$. The collection of affine functions $\left\{\mu_{i}(\cdot): i \in \mathbb{N}_{[1: N]}\right\}$ given via $(22)-(23)$, leads to the introduction of the dynamics of scaling factors taking the form of a simple affine, discrete-time autonomous system:

$$
\theta^{+}=M \theta+\alpha
$$

where $\theta$ is the vector of current scaling factors, $\theta^{+}$is the vector of successor scaling factors, and the $\theta$-transition matrix $M \in \mathbb{R}_{+}^{N \times N}$ as well as $\alpha$, the offset vector, are composed, respectively, from the scalars $\mu_{(i, j)} \in \mathbb{R}_{+},(i, j) \in$ $\mathbb{N}_{[1: N]} \times \mathbb{N}_{[1: N]}$ and the scalars $\alpha_{i}, i \in \mathbb{N}_{[1: N]}$ specified in (23). Evidently, the system-theoretic analysis of the affine dynamics of the scaling factors is simpler compared to the case of the exact, non-linear, dynamics and, hence, it further enhances computational practicability. Furthermore, this affine representation yields additional 
insights into the corresponding considerations; namely, the diagonal terms of the matrix $M$ can be directly linked to the stability properties of the local subsystems, while the off-diagonal characterize the physical interconnection effects. Obviously, the successor scaling factor $\theta^{+}$is composed from the two components, the first one being $M \theta$ which captures the effects of the physical interconnections, while the second one is $\alpha$ which represents the effect of the uncertainty induced from the disturbance sets $\mathbb{W}_{i}$. Clearly, by construction, it follows that for any $\theta \in \mathbb{R}_{+}^{N}$ and any $X=\left(X_{1}, X_{2}, \ldots, X_{N}\right) \in 2^{\mathbb{R}^{n_{1}}} \times 2^{\mathbb{R}^{n_{2}}} \times \ldots 2^{\mathbb{R}^{n_{N}}}$ such that, for all $i \in \mathbb{N}_{[1: N]}$, $X_{i} \subseteq \theta_{i} S_{i}$ it holds that $F_{i}\left(\left(X_{1}, X_{2}, \ldots, X_{N}\right), \mathbb{W}\right) \subseteq F_{i}\left(\left(\theta_{1} S_{1}, \theta_{2} S_{2}, \ldots, \theta_{N} S_{N}\right), \mathbb{W}\right) \subseteq \theta_{i}^{+} S_{i}$ with $\theta_{i}^{+}=\mu_{i}(\theta)$. Hence, the condition (20b) in the practical robust positive invariance notions of Definition 4.3 is ensured by considering the $\theta$-dynamics as specified in (24). In order to ensure the satisfaction of the condition (20a) it is necessary to restrict the values of the scaling factors $\theta$ to an adequate subset $\Theta$ of the set of constraint admissible scaling factors $\Theta_{0}$ given by:

$$
\Theta_{0}:=\left\{\theta \in \mathbb{R}_{+}^{N}: \forall i \in \mathbb{N}_{(N, i)}, \theta_{i} S_{i} \subseteq \mathbb{X}_{i}\right\}
$$

We note that the set $\Theta_{0}$ is, under Assumptions 2.1 and 4.1, a convex and non-empty compact subset of $\mathbb{R}_{+}^{N}$. In order to guarantee the non-emptiness of a suitable set $\Theta \subseteq \Theta_{0}$ as well as to ensure the satisfaction of the condition (20c) we provide the following set of natural sufficient conditions:

Assumption 4.2 (i) The matrix $M$ inducing the $\theta$-dynamics in $(24)$ is strictly stable, i.e. $\rho(M)<1$,

(ii) the unique fixed point of the equation $\theta=M \theta+\alpha$ say $\bar{\theta}$ is such that $\bar{\theta} \in \Theta_{0}$, and,

(iii) the set $\Theta \subseteq \mathbb{R}_{+}^{N}$ is a non-trivial convex and compact positively invariant set for the system (24) and constraint set (25), i.e. $\forall \theta \in \Theta \subseteq \Theta_{0}, M \theta+\alpha \in \Theta$.

Remark 4.1 We note that Assumption 4.2 $(i)$ is an usual requirement which is also utilized in the nominal case [12]. Assumption 4.2 $(\mathrm{ii})$ is also a reasonable condition since $\rho(M)<1$, so that the fixed point $\bar{\theta}=\sum_{k=0}^{\infty} M^{k} \alpha=$ $(I-M)^{-1} \alpha$ is a globally stable attractor for the $\theta$-dynamics specified in (24). Finally, the satisfaction of Assumption 4.2 (iii) is guaranteed under Assumptions 2.1, 4.2 $(i)$ and 4.2 (ii). In particular, the corresponding set $\Theta$ as specified in Assumption 4.2 (iii) can be obtained by the standard set iteration:

$$
\forall k \in \mathbb{N}, \Theta_{k+1}=M^{-1}\left(\{-\alpha\} \oplus \Theta_{k}\right) \bigcap \Theta_{0}
$$

where $M$ is specified in (24) and $\Theta_{0}$ given by (25). Under Assumptions 2.1, 4.2 $(i)$ and 4.2 (ii), the set iteration above results in a sequence of convex and compact sets $\left\{\Theta_{k}\right\}_{k \in \mathbb{N}}$ such that, for all $k \in \mathbb{N},\{\bar{\theta}\} \subseteq \Theta_{k+1} \subseteq \Theta_{k}$. In fact, the set sequence $\left\{\Theta_{k}\right\}_{k \in \mathbb{N}}$ admits the limit, say $\Theta_{\infty}$, with respect to the Hausdorff distance which is the maximal positively invariant set for the system (24) and constraint set (25). Furthermore, the standard results [8, 11] imply that the limit $\Theta_{\infty}$ of the set sequence $\left\{\Theta_{k}\right\}_{k \in \mathbb{N}}$ is, under Assumptions 2.1, 4.2 (i) and 4.2 (ii), finitely determined, i.e. there exists a finite integer $k^{*}$ such that $\Theta_{k^{*}}=\Theta_{k^{*}+1}=\Theta_{\infty}$; in addition, the set $\Theta_{\infty}$ is a polytopic subset of $\mathbb{R}_{+}^{N}$ which is guaranteed to contain the set $\left[0, \bar{\theta}_{1}\right] \times\left[0, \bar{\theta}_{2}\right] \times \ldots \times\left[0, \bar{\theta}_{N}\right]$ (here $\bar{\theta}_{i}$ are the coordinates of the corresponding fixed point $\left.\bar{\theta}=\left(\bar{\theta}_{1}, \bar{\theta}_{2}, \ldots \bar{\theta}_{N}\right)\right)$.

Utilizing the preceding construction and invoked assumptions we are able to offer our first main result, which verifies the existence of non-trivial robust positively invariant family of sets $\mathcal{S}(\mathbb{S}, \Theta)$ :

Proposition 4.2 Suppose Assumptions 2.1, 4.1 and 4.2 hold. Then the family of sets $\mathcal{S}(\mathbb{S}, \Theta)$ given by $(19)$ is a non-trivial, robust positively invariant family of sets.

\section{Convergence and Stability}

We now turn attention to the issues related to convergence and stability. As in the previous section, the $\theta$-dynamics given in (24) is employed to discuss the corresponding system-theoretic properties for set of $N$ subsystems (1) subject to the constraints (2). Under our assumptions, as already indicated, for any $\theta \in \Theta$ and any $X=\left(X_{1}, X_{2}, \ldots, X_{N}\right) \in$ $2^{\mathbb{R}^{n_{1}}} \times 2^{\mathbb{R}^{n_{2}}} \times \ldots 2^{\mathbb{R}^{n_{N}}}$ such that, for all $i \in \mathbb{N}_{[1: N]}, X_{i} \subseteq \theta_{i} S_{i}$ it holds that:

$$
\begin{aligned}
& F_{i}\left(\left(X_{1}, X_{2}, \ldots, X_{N}\right), \mathbb{W}\right) \subseteq F_{i}\left(\left(\theta_{1} S_{1}, \theta_{2} S_{2}, \ldots, \theta_{N} S_{N}\right), \mathbb{W}\right), \\
& F_{i}\left(\left(\theta_{1} S_{1}, \theta_{2} S_{2}, \ldots, \theta_{N} S_{N}\right), \mathbb{W}\right) \subseteq \theta_{i}^{+} S_{i}, \text { with, } \\
& \theta_{i}^{+}=\mu_{i}(\theta) \in \Theta .
\end{aligned}
$$

Motivated by this fact we proceed to demonstrate how the stability properties of the $\theta$-dynamics in (24) can be utilized to obtain guaranteed robust stability properties of the exact induced, independent set-dynamics in (15)-(16) as well as the original set of $N$ systems specified in (1). 


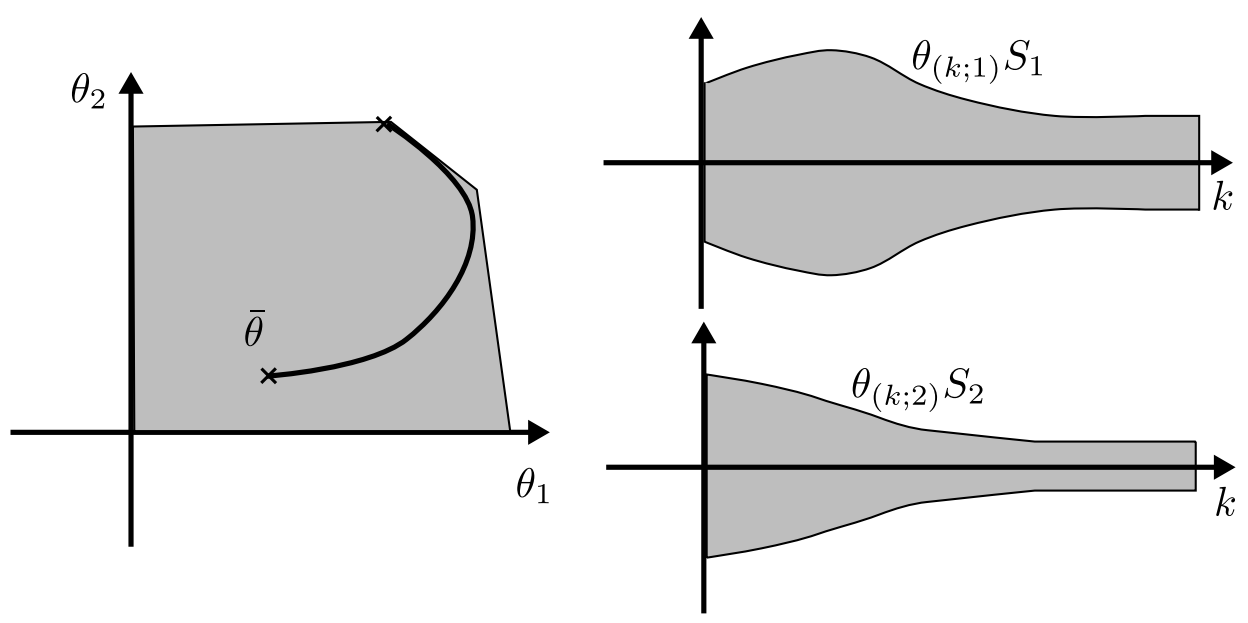

Figure 1: The illustration of the $\theta$-dynamics.

For the subsequent analysis, let $\boldsymbol{X}\left(X_{0}\right)$ denote, for any $X_{0}=\left(X_{(0 ; 1)}, X_{(0 ; 1)}, \ldots, X_{(0 ; N)}\right) \in 2^{\mathbb{R}^{n_{1}}} \times 2^{\mathbb{R}^{n_{2}}} \times \ldots 2^{\mathbb{R}^{n_{N}}}$, the sequence $\left\{X_{k}=\left(X_{(k ; 1)}, X_{(k ; 2)}, \cdots X_{(k ; N)}\right)\right\}_{k \in \mathbb{N}}$, generated by $(15)-(16)$, i.e. for all $k \in \mathbb{N}$ and all $i \in \mathbb{N}_{[1: N]}$,

$$
X_{(k+1 ; i)}=F_{i}\left(X_{k}, \mathbb{W}\right),
$$

where the maps $F_{i}(\cdot, \cdot), i \in \mathbb{N}_{[1: N]}$ are given by (16). Let also $\boldsymbol{Y}\left(Y_{0}\right)$ denote, for any $\theta_{0}=\left(\theta_{(0 ; 1)}, \theta_{(0 ; 2)}, \ldots, \theta_{(0 ; N)}\right) \in$ $\mathbb{R}_{+}^{N}$ and $Y_{0}=\left(\theta_{(0 ; 1)} S_{1}, \theta_{(0 ; 2)} S_{2}, \ldots, \theta_{(0 ; N)} S_{N}\right)$, the sequence $\left\{Y_{k}=\left(\theta_{(k ; 1)} S_{1}, \theta_{(k ; 2)} S_{2}, \cdots \theta_{(k ; N)} S_{N}\right)\right\}_{k \in \mathbb{N}}$, where for all $k \in \mathbb{N}$ and all $i \in \mathbb{N}_{[1: N]}, \theta_{k}=\left(\theta_{(k ; 1)}, \theta_{(k ; 2)}, \ldots, \theta_{(k ; N)}\right)$ and

$$
\theta_{k+1}=M \theta_{k}+\alpha
$$

Our next main result complements practical robust positive invariance properties established in Section 4 with the corresponding convergence properties:

Theorem 5.1 Suppose Assumptions 2.1, 4.1 and 4.2 hold. Consider the family of sets $\mathcal{S}(\mathbb{S}, \Theta)$ given by (19) and any sequence $\boldsymbol{Y}\left(Y_{0}\right)$ generated by (28) with $Y_{0} \in \mathcal{S}(\mathbb{S}, \Theta)$. Then for all $k \in \mathbb{N}$,

(i) $Y_{k} \in \mathcal{S}(\mathbb{S}, \Theta)$,

(ii)

$$
\sum_{i \in \mathbb{N}_{[1: N]}} H\left(L_{i}, \theta_{(k ; i)} S_{i}, \bar{\theta}_{i} S_{i}\right) \leq a^{k} b \sum_{i \in \mathbb{N}_{[1: N]}} H\left(L_{i}, \theta_{(0 ; i)} S_{i}, \bar{\theta}_{i} S_{i}\right)
$$

for some scalars $a \in[0,1)$ and $b \in(0, \infty)$, and,

(iii) $H\left(L_{i}, \theta_{(k ; i)} S_{i}, \bar{\theta}_{i} S_{i}\right) \rightarrow 0$ as $k \rightarrow \infty$, where $\bar{\theta}=\left(\bar{\theta}_{1}, \bar{\theta}_{2}, \ldots, \bar{\theta}_{N}\right)$ is the fixed point specified in Assumption 4.2(ii).

As illustrated in Figure 1, the invariance and stability properties of the $\theta$-dynamics yield in a transparent way the corresponding invariance and stability properties of the outer-bounding set-dynamics which generate sequences $\left\{Y_{k}=\left(\theta_{(k ; 1)} S_{1}, \theta_{(k ; 2)} S_{2}, \cdots \theta_{(k ; N) S_{N}}\right)\right\}_{k \in \mathbb{N}}$.

The relationships indicated in (26) allow for a direct utilization of Theorem 5.1 to discuss guaranteed invariance and convergence issues for the exact induced, independent set-dynamics in (15)-(16). Namely, while Theorem 5.1 yields convergence with respect to the Hausdorff distance, the guaranteed inclusions in (26) allow us to discuss the convergence for the exact induced, independent set-dynamics in (15)-(16) with respect to the Hausdorff upper semi-distance.

Corollary 5.1 Suppose Assumptions 2.1, 4.1 and 4.2 hold. Consider the family of sets $\mathcal{S}(\mathbb{S}, \Theta)$ given by (18) and any two sequences $\boldsymbol{X}\left(X_{0}\right), \boldsymbol{Y}\left(Y_{0}\right)$ generated by (27) and (28) with, for all $i \in \mathbb{N}_{[1: N]}, X_{(0 ; i)} \subseteq Y_{(0 ; i)}$ for some $Y_{0} \in \mathcal{S}(\mathbb{S}, \Theta)$. Then for, all $k \in \mathbb{N}$ and all $i \in \mathbb{N}_{[1: N]}$,

(i) $X_{(k ; i)} \subseteq Y_{(k ; i)}$,

(ii) $X_{(k ; i)} \subseteq \mathbb{X}_{i}$,

(iii) $h\left(L_{i}, X_{(k ; i)}, \bar{\theta}_{i} S_{i}\right) \rightarrow 0$ as $k \rightarrow \infty$, where $\bar{\theta}=\left(\bar{\theta}_{1}, \bar{\theta}_{2}, \ldots \bar{\theta}_{N}\right)$ is the fixed point specified in Assumption 4.2 (ii). 
Remark 5.1 Coming back to our initial problem specified in Section 2, stability and convergence properties are now easily discussed. Utilizing Theorem 5.1 and Corollary 5.1 we can analyze the behavior of the set of state sequences $\left\{x_{(k ; i)}\right\}_{k \in \mathbb{N}}, i \in \mathbb{N}_{[1: N]}$ of the $N$ physically, interconnected systems that satisfy, for all $i \in \mathbb{N}_{[1: N]}$ and $k \in \mathbb{N}$,

$$
x_{(k+1 ; i)} \in A_{(i, i)} x_{(k ; i)}+\sum_{j \in \mathbb{N}_{(N, i)}} A_{(i, j)} x_{(k ; j)} \oplus \mathbb{W}_{i},
$$

with given initial conditions $x_{(0 ; i)}, i \in \mathbb{N}_{[1: N]}$. As a consequence, we can also deduce from Theorem 5.1 and Corollary 5.1 that for all $k \in \mathbb{N}$ and all $i \in \mathbb{N}_{[1: N]}$, it holds that $x_{(k ; i)} \in X_{(k ; i)} \subseteq \theta_{(k ; i)} S_{i}$. More precisely, any set of state sequences $\left\{x_{(k ; i)}\right\}_{k \in \mathbb{N}}, i \in \mathbb{N}_{[1: N]}$ that satisfies (29) is such that, for all $k \in \mathbb{N}$ and all $i \in \mathbb{N}_{[1: N]}$, it holds that

$$
x_{(k ; i)} \in \theta_{(k ; i)} S_{i} \subseteq \mathbb{X}_{i}
$$

providing, of course, that $x_{(0 ; i)} \in \theta_{(0 ; i)} S_{i}, i \in \mathbb{N}_{[1: N]}$ and $\theta_{0}=\left(\theta_{(0 ; 1)}, \theta_{(0 ; 2)}, \ldots, \theta_{(0 ; N)}\right) \in \Theta$ and $\left\{\theta_{k}\right\}_{k \in \mathbb{N}}$ is generated via (28). Furthermore, we also have that, for all $k \in \mathbb{N}$ and all $i \in \mathbb{N}_{[1: N]}$, it holds that, $\sum_{i \in \mathbb{N}_{[1: N]}} h\left(L_{i},\left\{x_{(k ; i)}\right\}, \bar{\theta}_{i} S_{i}\right) \leq$ $\sum_{i \in \mathbb{N}_{[1: N]}} h\left(L_{i}, \theta_{(k, i)} S_{i}, \bar{\theta}_{i} S_{i}\right)$ and, hence,

$$
\sum_{i \in \mathbb{N}_{[1: N]}} h\left(L_{i},\left\{x_{(k ; i)}\right\}, \bar{\theta}_{i} S_{i}\right) \leq a^{k} b \sum_{i \in \mathbb{N}_{[1: N]}} h\left(L_{i}, \theta_{(0, i)} S_{i}, \bar{\theta}_{i} S_{i}\right)
$$

for some scalars $a \in[0,1)$ and $b \in(0, \infty)$ and where $\bar{\theta}=\left(\bar{\theta}_{1}, \bar{\theta}_{2}, \ldots \bar{\theta}_{N}\right)$ is the fixed point specified in Assumption 4.2 (ii). In turn, it also follows that $h\left(L_{i},\left\{x_{(k, i)}\right\}, \bar{\theta}_{i} S_{i}\right) \rightarrow 0$ as $k \rightarrow \infty$. In addition, the initialization of the subsystems (1) is also simplified, since each individual subsystem does not need the exact knowledge of the initial states of the other subsystems; in other words the only requirement for the safe and independent operation of the interconnected systems specified in (1) under uncertainty is the condition that $x_{(0 ; i)} \in \theta_{(0 ; i)} S_{i}$, with $\theta_{0}=\left(\theta_{(0 ; 1)}, \theta_{(0 ; 2)}, \ldots, \theta_{(0 ; N)}\right) \in \Theta$, for all $i \in \mathbb{N}_{[1: N]}$.

\section{Computational Remarks}

In this section we consider the case when the state and disturbance constraint sets are, respectively, $P C$ - and $C$-polytopic sets and are given, for all $i \in \mathbb{N}_{[1: N]}$, via their irreducible representations:

$$
\begin{aligned}
& \mathbb{X}_{i}:=\left\{x_{i} \in \mathbb{R}^{n_{i}}: \forall j \in \mathbb{N}_{\left[1: q_{i}\right]}, l_{(i, j)}^{T} x_{i} \leq 1\right\}, \text { and } \\
& \mathbb{W}_{i}:=\left\{w_{i} \in \mathbb{R}^{n_{i}}: \forall k \in \mathbb{N}_{\left[1: r_{i}\right]}, m_{(i, k)}^{T} w_{i} \leq t_{(i, k)}\right\},
\end{aligned}
$$

where, for all $j \in \mathbb{N}_{\left[1: q_{i}\right]}$ and $k \in \mathbb{N}_{\left[1: r_{i}\right]}, l_{(i, j)} \in \mathbb{R}^{n_{i}}, m_{(i, k)} \in \mathbb{R}^{n_{i}}$ and $t_{(i, k)} \in \mathbb{R}_{+}$, respectively, for all $i \in \mathbb{N}_{[1: N]}$.

With the requirement of the independent operation and the objective to detect and compute locally the candidate sets $S_{i}, i \in \mathbb{N}_{[1: N]}$ in mind, a meaningful way is to employ the so-called $\lambda$-contractive sets [7]. Namely, the underlying idea is to consider, for all $i \in \mathbb{N}_{[1: N]}$, the set of $N$ subsystems whose dynamics and constraints take the form:

$$
\begin{aligned}
& x_{i}^{+}=A_{(i, i)} x_{i}+w_{i}, \text { and } \\
& x_{i} \in \mathbb{X}_{i} \text { and } w_{i} \in \mathbb{W}_{i} .
\end{aligned}
$$

The interconnection terms $\sum_{j \in \mathbb{N}_{(N, i)}} A_{(i, j)} x_{j}$ are neglected in (31), but their effect is compensated for indirectly by computing the maximal or just $\lambda$-contractive sets $S_{i}, i \in \mathbb{N}_{[1: N]}$. The corresponding $\lambda$-contractive sets $S_{i}, i \in \mathbb{N}_{[1: N]}$ are such that:

$$
A_{(i, i)} S_{i} \oplus \mathbb{W}_{i} \subseteq \lambda_{i} S_{i} \text { and } S_{i} \subseteq \mathbb{X}_{i}
$$

where the contraction factors $\lambda_{i}, i \in \mathbb{N}_{[1: N]}$ satisfy, for all $i \in \mathbb{N}_{[1: N]}, \lambda_{i} \in[0,1)$. Hence, the value of contraction factors $\lambda_{i}$ should be made as small as possible in order to increase the size of sets $\left(1-\lambda_{i}\right) S_{i}$ which can be used to compensate for the effect of the neglected interconnection terms $\sum_{j \in \mathbb{N}_{(N, i)}} A_{(i, j)} x_{j}$. The computation of the $\lambda$ contractive sets $S_{i}, i \in \mathbb{N}_{[1: N]}$ for the subsystems and constraints sets can be realized efficiently by employing the standard methods [7] or more recent results on the parameterized $\lambda$-contractive sets $[15,16]$. It is worth pointing out that sufficient conditions for the existence of the corresponding $\lambda$-contractive sets, reduce to the conditions that, for all $i \in \mathbb{N}_{[1: N]}$, the matrices $A_{(i, i)}$ are strictly stable (i.e. $\rho\left(A_{(i, i)}\right)<1$ ) and that the minimal robust positively invariant sets $[8,13,10,1]$ given, for all $i \in \mathbb{N}_{[1: N]}$, by $X_{(\infty, i)}=\bigoplus_{k=0}^{\infty} A_{(i, i)}^{k} \mathbb{W}_{i}$ satisfy, for all $i \in \mathbb{N}_{[1: N]}$, $X_{(\infty, i)} \subseteq$ interior $\left(\mathbb{X}_{i}\right)$. In fact, under these conditions it follows that the maximal $\lambda$-contractive sets are computable in finite time; furthermore, in this case, they are $P C$-polytopic sets and, hence, admit irreducible representations given, for all $i \in \mathbb{N}_{[1: N]}$, by:

$$
S_{i}:=\left\{s_{i} \in \mathbb{R}^{n_{i}}: \forall p \in \mathbb{N}_{\left[1: \tau_{i}\right]}, \phi_{(i, p)}^{T} s_{i} \leq 1\right\}
$$


where, for all $p \in \mathbb{N}_{\left[1: \tau_{i}\right]}, \phi_{(i, p)} \in \mathbb{R}^{n_{i}}$. The computation of the scalars $\mu_{(i, j)}$ forming the matrix $M$ is efficiently done by using the basic properties of the support function [14,17]. Namely, the set inclusions $A_{(i, j)} S_{i} \subseteq \mu_{(i, j)} S_{i}$ are satisfied, for all $i \in \mathbb{N}_{[1: N]}$ and all $j \in \mathbb{N}_{[1: N]}$, if and only if, for all $p \in \mathbb{N}_{\left[1: \tau_{i}\right]}$, it holds that $s\left(S_{j}, A_{(i, j)}^{T} \phi_{(i, p)}\right) \leq \mu_{(i, j)}$. In turn, it follows that, for all $i \in \mathbb{N}_{[1: N]}$ and all $j \in \mathbb{N}_{[1: N]}$, the corresponding scalars $\mu_{(i, j)}$ are given by:

$$
\mu_{(i, j)}:=\max _{p}\left\{s\left(S_{j}, A_{(i, j)}^{T} \phi_{(i, p)}\right): p \in \mathbb{N}_{\left[1: \tau_{i}\right]}\right\}
$$

and can be computed by solving a sequence of well-defined linear programs. Likewise, the set inclusions $W_{i} \subseteq \alpha_{i} S_{i}$ are satisfied, for all $i \in \mathbb{N}_{[1: N]}$, if and only if, for all $p \in \mathbb{N}_{\left[1: \tau_{i}\right]}$, it holds that $s\left(\mathbb{W}_{i}, \phi_{(i, p)}\right) \leq \alpha_{i}$. Hence, for all $i \in \mathbb{N}_{[1: N]}$, the corresponding scalars $\alpha_{i}$ are given by:

$$
\alpha_{i}:=\max _{p}\left\{s\left(\mathbb{W}_{i}, \phi_{(i, p)}\right): p \in \mathbb{N}_{\left[1: \tau_{i}\right]}\right\}
$$

and, as above, can be computed by solving a sequence of well-defined linear programs. Finally, the set $\Theta_{0}$ specified in (25) takes the polytopic form given by:

$$
\Theta_{0}=\left\{\theta \in \mathbb{R}_{+}^{N}: \forall i \in \mathbb{N}_{[1: N]}, \theta_{i} \leq \max _{j \in \mathbb{N}_{\left[1: q_{i}\right]}} s\left(S_{i}, l_{(i, j)}\right)\right\}
$$

and, consequently, the polytopic set $\Theta$ (see Assumption $4.2($ iii $)$ ) can be computed as indicated in Remark 4.1 .

\subsection{Illustrative Example}

We consider a nine dimensional, discrete-time linear system, $x^{+}=A x+w$, where

$$
A=\left(\begin{array}{ccc|ccc|ccc}
0.1 & 0.3 & 0 & -0.1 & 0 & 0 & 0.1 & 0.1 & 0 \\
0.1 & 0 & 0.3 & 0 & 0 & 0 & 0 & 0 & 0.1 \\
-0.3 & 0.3 & 0.3 & 0 & 0 & 0.1 & 0 & 0 & 0 \\
\hline 0 & 0 & 0 & 0 & 0.5 & 0 & -0.1 & 0.1 & 0 \\
0 & 0.1 & 0 & 0 & 0 & 0.5 & 0 & 0 & 0 \\
0 & 0 & 0.1 & -0.1 & 0.2 & 0.3 & 0 & 0 & 0.1 \\
\hline 0.1 & 0 & 0 & 0 & 0 & 0 & 0.1 & 0.1 & 0 \\
0.1 & 0.1 & 0 & 0 & 0 & 0 & 0 & 0 & 0.1 \\
0 & 0 & 0.1 & 0 & 0 & 0.1 & 0.3 & -0.1 & 0.2
\end{array}\right),
$$

and treat it as three interconnected systems with the matrices $A_{(i, j)}$ as indicated by the partition of the matrix $A$. The corresponding state and disturbance constraint sets are:

$$
\begin{aligned}
& \mathbb{X}_{1}=\mathbb{X}_{2}=\mathbb{X}_{3}=\left\{x \in \mathbb{R}^{3}:|x|_{\infty} \leq 1\right\}, \text { and } \\
& \mathbb{W}_{1}=\mathbb{W}_{2}=\mathbb{W}_{3}=\left\{w \in \mathbb{R}^{3}:|w|_{\infty} \leq 0.1\right\}
\end{aligned}
$$

The collection of sets $\left\{S_{1}, S_{2}, S_{2}\right\}$ is obtained by computing $P C$-polytopic, $\lambda$-contractive sets, for the subsystems $x_{i}^{+}=A_{(i, i)} x_{i}$, for $i \in\{1,2,3\}$, with the contraction factors $(0.595,0.589,0.459)$. The $\lambda$-contractive sets $S_{i}$, for $i \in\{1,2,3\}$ are shown in Figure 2. The matrix $M$ and vector $\alpha$ are computed by using relations (34) and (35); the matrix $M$ is strictly stable. The set $\Theta_{0}$ is given by $\Theta_{0}=\left\{\theta \in \mathbb{R}_{+}^{3}:\left|\theta_{1}\right| \leq 1.202,\left|\theta_{2}\right| \leq 1.2045,\left|\theta_{3}\right| \leq 1.2786\right\}$ while the set $\Theta$ is computed according to Remark 4.1 and depicted in the upper part of Figure 2. The corresponding fixed point $\bar{\theta}$ is, in this case, given by $\bar{\theta}=(0.8986,0.7444,0.6891)$ and it lies in the interior of the set $\Theta \subseteq \Theta_{0}$. Hence, all our assumptions are satisfied. We select $\theta_{0}=(0.6328,1.0097,1.0469)$, which is an extreme point of the set $\Theta$, and simulate several sets of state trajectories, with initial conditions being equal to the extreme points of the sets $\theta_{(0 ; i)} S_{i}$, for $i=\{1,2,3\}$.

As a comparison, in Figure 2 the set of state trajectories for the nominal case, i.e. $\mathbb{W}_{i}=\{0\}$ for $i=\{1,2,3\}$, and the robust case are both shown. As expected in view of Theorem 5.1 and Corollary 5.1, in both cases the state constraints are satisfied, and the trajectories converge to the sets $\bar{\theta}_{i} S_{i}$, for $i \in\{1,2,3\}$ in a stable fashion (while for the nominal case, convergence to and stability of the origin is guaranteed).

\section{Conclusions}

In this paper, we discussed exact and practical robust positive invariance notions for physically interconnected, discrete-time systems. For computational tractability, we provided a relaxation of the exact notions, by employing a suitably parametrized family of sets. It was shown, that under mild and natural assumptions, the derived notions allow for the safe, independent operation for each of the physically interconnected subsystems, regardless of the presence of additive bounded disturbances. 


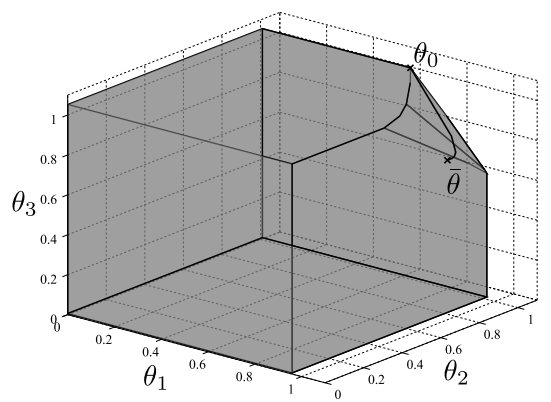

(a) System $\theta_{k+1}=M \theta_{k}$ and set $\Theta$

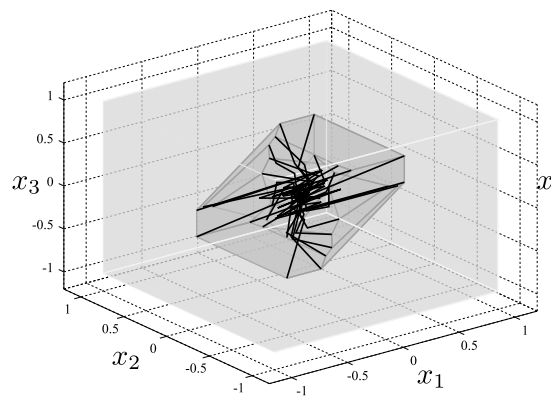

(b) Subsystem 1: Nominal case

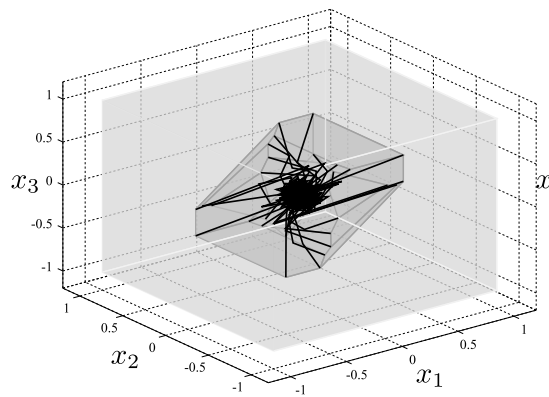

(e) Subsystem 1: Robust case

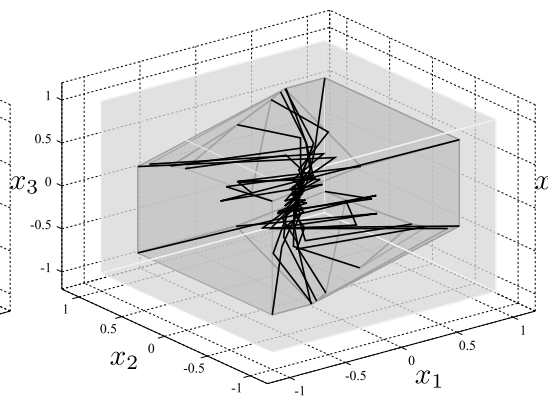

(c) Subsystem 2: Nominal case

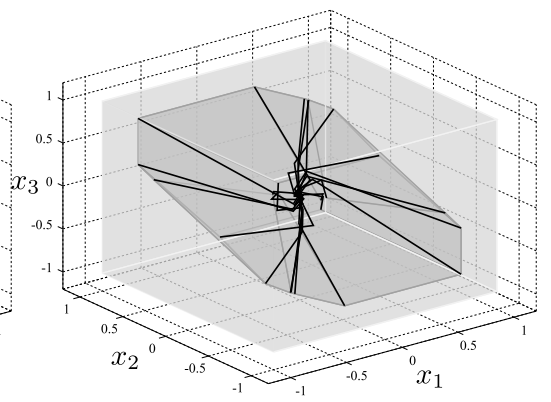

(d) Subsystem 3: Nominal case

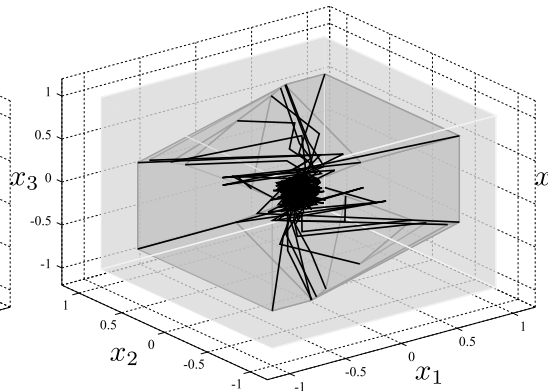

(f) Subsystem 2: Robust case

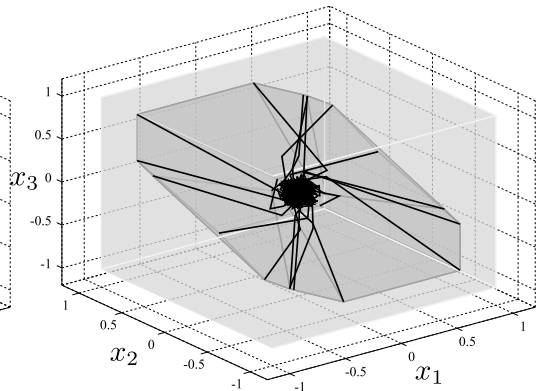

(g) Subsystem 3: Robust case

Figure 2: $\theta$-dynamics, with initial condition $\theta_{0}$, and sets of sample trajectories initialized in the sets $\theta_{(0 ; i)} S_{i}$, with the constraint sets $\mathbb{X}_{i}$ for $i \in\{1,2,3\}$.

\section{References}

[1] Zvi Artstein and Saša V. Raković. Feedback and Invariance under Uncertainty via Set Iterates. Automatica, $44(2): 520-525,2008$.

[2] Zvi Artstein and Saša V. Raković. Set Invariance under Output Feedback: A Set-Dynamics Approach. International Journal of Systems Science, 44(2):520-525, 2010.

[3] J.P. Aubin. Viability Theory. Birkhäuser, Boston, 1991.

[4] L. Bakule. Decentralized control: An overview. Ann. Rev. in Contr., 32(1):87-98, 2008.

[5] R. Bellman. Vector lyapunov functions. SIAM J. Contr., 1:32-34, 1962.

[6] F. Blanchini. Set invariance in control. Automatica, 35(11):1747-1767, 1999.

[7] F. Blanchini and S. Miani. Set-theoretic methods in control. Birkhäuser, Boston, 2008.

[8] I. Kolmanovsky and E.G. Gilbert. Theory and computation of disturbance invariant sets for discrete-time linear systems. Mathematical Problems in Engineering, 4(4):317-363, 1998.

[9] V. Lakshmikantham, V.M. Matrosov, and S. Sivasundaram. Vector Lyapunov functions and stability analysis of nonlinear systems. Kluwer Academic Publishers, Dordrecht, Boston, London, 1991.

[10] S. V. Raković. Minkowski Algebra and Banach Contraction Principle in Set Invariance for Linear Discrete Time Systems. In Proceedings of 46 th IEEE Conference on Decision and Control, CDC 2007, New Orleans, LA, USA, December 2007. 
[11] S. V. Raković and M. Fiacchini. Invariant Approximations of the Maximal Invariant Set of "Encircling the Square". In Proceedings of the 17th IFAC World Congress IFAC 2008, Seoul, Korea, 2008.

[12] S. V. Raković, B. Kern, and R. Findeisen. Practical set invariance for decentralized discrete time systems. In Proc. of the IEEE Conf. on Dec. and Contr., CDC'10, Atlanta, USA, 2010. Submitted.

[13] S. V. Raković, E. C. Kerrigan, K. I. Kouramas, and D. Q. Mayne. Invariant approximations of the minimal robustly positively invariant sets. IEEE Transactions on Automatic Control, 50(3):406-410, 2005.

[14] R.T. Rockafellar. Convex Analysis. Princeton University Press, 1970.

[15] S. V. Raković and M. Barić. Local Control Lyapunov Functions for Constrained Linear Discrete-Time Systems: The Minkowski Algebra Approach. IEEE Transactions on Automatic Control, 54(11):2686-2692, 2009.

[16] S. V. Raković and M. Barić. Parameterized Robust Control Invariant Sets for Linear Systems: Theoretical Advances and Computational Remarks. IEEE Transactions on Automatic Control, 55(7):1599-1614, 2010.

[17] R. Schneider. Convex bodies: the Brunn-Minkowski theory. Cambridge Univ Pr, 1993.

[18] D.D. Šiljak. Large-scale dynamic systems: stability and structure. North Holland, 1978.

[19] D. D. Šiljak and A. I. Zečević. Control of large-scale systems: beyond decentralized feedback. Ann. Rev. in Contr., 29:169-179, 2005.

\section{A Proofs}

\section{Proof of Proposition 4.1}

Convexity: Pick any $\lambda$ such that $0 \leq \lambda \leq 1$ and any $\theta^{1} \in \mathbb{R}_{+}^{N}, \theta^{2} \in \mathbb{R}_{+}^{N}$. By properties of Minkowski set addition and definition of $\mu_{i}^{e}(\cdot)$ the relations

$\bigoplus_{j \in \mathbb{N}_{[1: N]}}\left(\lambda \theta_{j}^{1}+(1-\lambda) \theta_{j}^{2}\right) A_{(i, j)} S_{j} \oplus \mathbb{W}_{i}=\bigoplus_{j \in \mathbb{N}_{[1: N]}} \lambda \theta_{j}^{1} A_{(i, j)} S_{j} \oplus \bigoplus_{j \in \mathbb{N}_{[1: N]}}(1-\lambda) \theta_{j}^{2} A_{(i, j)} S_{j} \oplus \mathbb{W}_{i} \subseteq \mu_{i}^{e}\left(\lambda \theta^{1}+(1-\lambda) \theta^{2}\right) S_{i}$

hold true and, similarly, we also have:

$$
\bigoplus_{j \in \mathbb{N}_{[1: N]}} \lambda \theta_{j}^{1} A_{(i, j)} S_{j} \oplus \lambda \mathbb{W}_{i}=\lambda\left(\bigoplus_{j \in \mathbb{N}_{[1: N]}} \theta_{j}^{1} A_{(i, j)} S_{j} \oplus \mathbb{W}_{i}\right) \subseteq \lambda \mu_{i}^{e}\left(\theta^{1}\right) S_{i}
$$

and, by the same token,

$$
\bigoplus_{j \in \mathbb{N}_{[1: N]}}(1-\lambda) \theta_{j}^{2} A_{(i, j)} S_{j} \oplus(1-\lambda) \mathbb{W}_{i} \subseteq(1-\lambda) \mu_{i}^{e}\left(\theta^{2}\right) S_{i}
$$

By assumption $\mathbb{W}_{i}$ are convex and compact sets and, hence, $\mathbb{W}_{i}=(1-\lambda) \mathbb{W}_{i} \oplus \lambda \mathbb{W}_{i}$ so that by utilizing (38) and (39) we obtain:

$$
\bigoplus_{j \in \mathbb{N}_{[1: N]}} \lambda \theta_{j}^{1} A_{(i, j)} S_{j} \oplus \bigoplus_{j \in \mathbb{N}_{[1: N]}}(1-\lambda) \theta_{j}^{2} A_{(i, j)} S_{j} \oplus \mathbb{W}_{i} \subseteq \lambda \mu_{i}^{e}\left(\theta^{1}\right) S_{i} \oplus(1-\lambda) \mu_{i}^{e}\left(\theta^{2}\right) S_{i}=\left((1-\lambda) \mu_{i}^{e}\left(\theta^{2}\right)+\lambda \mu_{i}^{e}\left(\theta^{1}\right)\right) S_{i} .
$$

Hence, in view of (37) and (40), by optimality of $\mu_{i}^{e}\left(\lambda \theta^{1}+(1-\lambda) \theta^{2}\right)$, yields that $\mu_{i}^{e}\left(\lambda \theta^{1}+(1-\lambda) \theta^{2}\right) \leq \lambda \mu_{i}^{e}\left(\theta^{1}\right)+$ $(1-\lambda) \mu_{i}^{e}\left(\theta^{2}\right)$ verifying convexity of $\mu_{i}^{e}(\cdot)$.

Continuity: The continuity of any $\mu_{i}^{e}(\cdot), i \in \mathbb{N}_{[1: N]}$ follow from its definition in (21) due to Assumptions 2.1 and 4.1 .

\section{Proof of Proposition 4.2}

Let $\theta=\left(\theta_{1}, \theta_{2}, \ldots \theta_{N}\right) \in \Theta$. By construction, relations (22)-(23) imply that, for all $i \in \mathbb{N}_{[1: N]}$, we have

$$
F_{i}\left(\left(\theta_{1} S_{1}, \theta_{2} S_{2}, \ldots, \theta_{N} S_{N}\right), \mathbb{W}\right)=\bigoplus_{j \in \mathbb{N}_{[1: N]}} \theta_{j} A_{(i, j)} S_{j} \oplus \mathbb{W}_{i} \subseteq \bigoplus_{j \in \mathbb{N}_{[1: N]}} \theta_{j} \mu_{(i, j)} S_{i} \oplus \alpha_{i} S_{i}=\left(\sum_{j \in \mathbb{N}_{[1: N]}} \theta_{j} \mu_{(i, j)}+\alpha_{i}\right) S_{i}=\theta_{i}^{+} S_{i}
$$

By Assumption 4.2, $\Theta$ is a robust positively invariant set, i.e. $\forall \theta \in \Theta, M \theta+\alpha \in \Theta$, so that $\theta^{+}=\left(\theta_{1}^{+}, \theta_{2}^{+}, \ldots \theta_{N}^{+}\right) \in \Theta$ and, hence, $\left(\theta_{1}^{+} S_{1}, \theta_{2}^{+} S_{2}, \ldots \theta_{N}^{+} S_{N}\right) \in \mathcal{S}(\mathbb{S}, \Theta)$. By Assumption $4.2, \Theta \subseteq \Theta_{0}$ and, hence, for any $\theta=\left(\theta_{1}, \theta_{2}, \ldots \theta_{N}\right) \in$ $\Theta$ and all $i \in \mathbb{N}_{[1: N]}$, it holds that $\theta_{i} S_{i} \subseteq \mathbb{X}_{i}$. The fact that $\mathcal{S}(\mathbb{S}, \Theta)$ is non-trivial follows from Assumption $4.2(i i i)$ under which $\Theta$ is a non-trivial set (the proof that non-trivial robust positively invariant set $\Theta$ of Assumption 4.2 (iii) exists under Assumptions 2.1, $4.2(i)$ and ( $i i)$ can be constructed analogously as in [12] and is therefore omitted). 


\section{Proof of Theorem 5.1}

(i) Since $\mathcal{S}(\mathbb{S}, \Theta)$ is a robust positively invariant family of sets, we have that $Y_{k} \in \mathcal{S}(\mathbb{S}, \Theta)$ implies $Y_{k+1} \in \mathcal{S}(\mathbb{S}, \Theta)$. But $Y_{0} \in \mathcal{S}(\mathbb{S}, \Theta)$ and the claim follows by induction.

(ii) By assumption, for all $i \in \mathbb{N}_{[1: N]}, S_{i}$ are proper $C$-sets and $L_{i}$ are proper symmetric $C$-sets. Thus, there exist scalars $\eta_{1} \in(0, \infty), \eta_{2} \in(0, \infty)$, such that $\eta_{1} L_{i} \subseteq S_{i} \subseteq \eta_{2} L_{i}$. If $\bar{\theta}_{i} \leq \theta_{i}$, then trivially $\bar{\theta}_{i} S_{i} \subseteq \theta_{i} S_{i}$ and

$$
\bar{\theta}_{i} S_{i} \oplus \eta_{1}\left(\theta_{i}-\bar{\theta}_{i}\right) L_{i} \subseteq \bar{\theta}_{i} S \oplus\left(\theta_{i}-\bar{\theta}_{i}\right) S_{i}=\theta_{i} S_{i} \subseteq \bar{\theta}_{i} S_{i} \oplus \eta_{2}\left(\theta_{i}-\bar{\theta}_{i}\right) L_{i} .
$$

Similarly, if $\theta_{i} \leq \bar{\theta}_{i}$ then $\theta_{i} S_{i} \subseteq \bar{\theta}_{i} S_{i}$ and

$$
\theta_{i} S_{i} \oplus \eta_{1}\left(\theta_{i}-\bar{\theta}_{i}\right) L_{i}=\theta_{i} S_{i} \oplus \eta_{1}\left(\bar{\theta}_{i}-\theta_{i}\right) L_{i} \subseteq \theta_{i} S_{i} \oplus\left(\bar{\theta}_{i}-\theta_{i}\right) S_{i}=\bar{\theta}_{i} S_{i} \subseteq \theta_{i} S_{i} \oplus \eta_{2}\left(\bar{\theta}_{i}-\theta_{i}\right) L_{i}=\theta_{i} S_{i} \oplus \eta_{2}\left(\theta_{i}-\bar{\theta}_{i}\right) L_{i}
$$

By the definition of the Hausdorff distance it follows that:

$$
\eta_{1}\left|\theta_{i}-\bar{\theta}_{i}\right| \leq H\left(L_{i}, \theta_{i} S_{i}, \bar{\theta}_{i} S_{i}\right) \leq \eta_{2}\left|\theta_{i}-\bar{\theta}_{i}\right|
$$

Summing over $i \in \mathbb{N}_{[1: N]}$ we obtain:

$$
\eta_{1} \sum_{i \in \mathbb{N}_{[1: N]}}\left|\theta_{i}-\bar{\theta}_{i}\right| \leq \sum_{i \in \mathbb{N}_{[1: N]}} H\left(L_{i}, \theta_{i} S_{i}, \bar{\theta}_{i} S_{i}\right) \leq \eta_{2} \sum_{i \in \mathbb{N}_{[1: N]}}\left|\theta_{i}-\bar{\theta}_{i}\right| .
$$

Since $\rho(M)<1$, there exist scalars $\tilde{a} \in[0,1)$ and $\tilde{b} \in(0, \infty)$ such that:

$$
\left|\theta_{k}-\bar{\theta}\right|_{L} \leq \tilde{a}^{k} \tilde{b}\left|\theta_{0}-\bar{\theta}\right|_{L}
$$

Furthermore, there exists scalars $\eta_{3} \in(0, \infty)$ and $\eta_{4} \in(0, \infty)$ such that:

$$
\eta_{3}|\theta-\bar{\theta}|_{L} \leq \sum_{i \in \mathbb{N}_{[1: N]}} H\left(L_{i}, \theta_{i} S_{i}, \bar{\theta}_{i} S_{i}\right) \leq \eta_{4}|\theta-\bar{\theta}|_{L}
$$

Using the preceding relations, we can always find scalars $a \in[0,1)$ and $b \in(0, \infty)$ such that

$$
\sum_{i \in \mathbb{N}_{[1: N]}} H\left(L_{i}, \theta_{(k, i)} S_{i}, \bar{\theta}_{i} S_{i}\right) \leq a^{k} b \sum_{i \in \mathbb{N}_{[1: N]}} H\left(L_{i}, \theta_{(0, i)} S_{i}, \bar{\theta}_{i} S_{i}\right)
$$

(iii) By (ii), $\sum_{i \in \mathbb{N}_{[1: N]}} H\left(L_{i}, \theta_{(k, i)} S_{i}, \bar{\theta}_{i} S_{i}\right) \rightarrow 0$ as $k \rightarrow \infty$ so that, $\forall i \in \mathbb{N}_{[1: N]}, H\left(L_{i}, \theta_{(k, i)} S_{i}, \bar{\theta}_{i} S_{i}\right) \rightarrow 0$ as $k \rightarrow \infty$.

\section{Proof of Corollary 5.1}

(i) Follows by induction since $F\left(X_{(k-1 ; i)}, \mathbb{W}_{i}\right)=X_{(k ; i)} \subseteq F\left(Y_{(k-1 ; i)}, \mathbb{W}_{i}\right)=Y_{(k ; i)} \in \mathcal{S}(\mathbb{S}, \Theta)$ and $X_{(0 ; i)} \subseteq Y_{(0 ; i)}$. (ii) and (iii) follow immediately from (i) and Theorem 5.1 (iii). 